\title{
2. Coopération avec les pays en développement
}

\section{Catherine Schümperli Younossian et Gérard Perroulaz}

\section{OpenEdition \\ Journals}

Édition électronique

URL : http://journals.openedition.org/aspd/420

DOI : $10.4000 /$ aspd.420

ISSN : 1663-9669

\section{Éditeur}

Institut de hautes études internationales et du développement

\section{Édition imprimée}

Date de publication : 1 avril 2005

Pagination : 15-34

ISSN : 1660-5934

\section{Référence électronique}

Catherine Schümperli Younossian et Gérard Perroulaz, « 2. Coopération avec les pays en

développement », Annuaire suisse de politique de développement [En ligne], 24-1 | 2005, mis en ligne le 01 mars 2010, consulté le 10 décembre 2020. URL : http://journals.openedition.org/aspd/420 ; DOI https://doi.org/10.4000/aspd.420 


\section{Coopération avec les pays en développement*}

$S$ UR LE PLAN INTERNATIONAL, la coopération au développement aura été marquée par un débat controversé sur l'opportunité d'inscrire la coopération au développement dans une optique de prévention contre le terrorisme. Si les acteurs gouvernementaux, dans le cadre du CAD, ont soutenu cette approche, de nombreux réseaux d'ONG ont mis en garde contre cette nouvelle orientation de l'aide.

En 2003, le niveau international de l'aide publique au développement (APD) a atteint 69 milliards de dollars, un montant jamais atteint précédemment. Les montants de l'APD suisse sont également élevés en 2003 (1748 millions de francs, soit 0,39\% du RNB). Cette hausse est toutefois surtout due à la notification différée des contributions à AID-13 ainsi qu'à des adaptations statistiques

En 2004, les deux agences en charge de la coopération au développement de la Suisse, la Direction du développement et de la coopération (DDC) et la division "Coopération économique au développement»du seco, ont publié des documents d'orientation stratégique en matière de lutte contre la pauvreté. A noter l'entrée en vigueur pour chacun des offices de leurs nouveaux crédits-cadre. La coopération multilatérale, gérée conjointement par la DDC et le seco, a également fait l'objet d'un document de stratégie conjointe.

En automne 2004, et suite à un long processus interne, la Communauté de travail des auvres d'entraide a rendu publiques ses lignes directrices en matière de politique de développement, ainsi que sa stratégie de travail pour les années à venir.

\subsection{Comité d'aide au développement de l'OCDE et Reality of Aid}

De nombreux acteurs participent à la définition des stratégies de développement, tant au niveau gouvernemental que parmi les acteurs de la société civile. Cette section présente deux «espaces » d'échanges et de dialogues; 1'un est officiel et gouvernemental: le Comité d'aide au développement de l'OCDE; le second, Reality of Aid, se compose de réseaux d'ONG du Sud comme du Nord, qui, entre autres objectifs, tentent de faire émerger une vision commune au sein des ONG pour la défense d'objectifs prioritaires à soutenir en matière de politiques de développement. En 2003 et 2004, le lien entre «terrorisme et développement» a fait l'objet de nombreux débats et controverses, succinctement présentés ci-dessous.

\subsubsection{Comité d'aide au développement de I'OCDE}

Le Comité d'aide au développement (CAD) de l'OCDE est le principal organe au sein duquel les agences de coopération des pays membres coordonnent leurs activités en vue d'accroître l'efficacité de leurs efforts.

* Par Catherine Schümperli Younossian, chargée de recherche à l'iuéd, avec la collaboration de Gérard Perroulaz, chargé de recherche à l'iuéd, pour la partie 2.4 . 
En vertu de son mandat, qui n'a pas changé depuis sa création en 1961, le CAD a «pour mission d'encourager des efforts coordonnés, intégrés et efficaces, assortis de moyens financiers suffisants, au niveau international en faveur d'un développement économique et social durable. Reconnaissant que ce sont les pays en développement eux-mêmes qui sont responsables, en dernier ressort, de leur propre développement, le CAD s'attache avant tout à voir comment la coopération internationale peut contribuer à renforcer la capacité de ces derniers de participer à l'économie mondiale et celle de leurs citoyens d'échapper à la pauvreté ${ }^{1}$.

\section{$\square$ Les activités du CAD}

\section{Un lieu de dialogue}

Le CAD tient chaque année une réunion de haut niveau, à laquelle les membres sont représentés par leur ministre de la Coopération au développement ou les responsables de leur organisme d'aide; participent également à ces rencontres des représentants des agences multilatérales de développement (FMI, BM, PNUD). Chaque année est en outre organisée une réunion au niveau des hauts fonctionnaires, qui passent en revue les travaux du comité sur les questions d'actualité. Les réunions ordinaires rassemblent les délégués des pays membres basés à Paris et des fonctionnaires venant des capitales. Le CAD organise également, à intervalles réguliers, des «forums des partenaires au développement» sur des sujets spécifiques, auxquels les pays partenaires sont largement représentés. Ces espaces de dialogue permettent aux membres d'échanger des données d'expériences et de parvenir à un consensus international sur les questions d'orientation de l'action et de gestion.

Accroître l'efficacité de l'aide a constitué le thème central de la réunion annuelle, les 15 et 16 avril 2004, des ministres de la Coopération au développement. Les ministres ont examiné les possibilités d'une coordination plus étroite entre donateurs et bénéficiaires, notamment en vue d'alléger la charge administrative de l'aide.

\section{Les examens par les pairs}

Dans le cadre de ces examens, qui ont habituellement lieu tous les quatre ans, le CAD étudie comment les orientations qu'il préconise sont appliquées dans le programme de chaque membre, analyse la façon dont le programme est géré, apprécie la cohérence des politiques suivies dans d'autres domaines avec les objectifs poursuivis en matière de développement, et retrace l'évolution du volume des ressources et de leur répartition. Par cette procédure, le CAD vise à encourager une amélioration permanente des efforts déployés par les membres dans tous les domaines de la coopération au développement. Les résultats de ces évaluations font 1'objet de rapports publics².

En 2005, la Suisse devra à nouveau se soumettre à un examen périodique du CAD. Dans cette perspective, les efforts déployés par la Suisse pour augmenter

1 CAD (OCDE), Coopération pour le développement. Rapport annuel 2003, Les dossiers du CAD, vol. 5, no 1, Paris, OCDE, 2004, p. 120.

2 Les rapports d'évaluation sont disponibles sur le site du CAD, <www.oecd.org/cad $>$, sous la rubrique «Examen par les pairs des membres du CAD». 
l'efficacité de l'aide, améliorer la cohérence politique et renforcer la coordination des activités entre pays donateurs restent déterminants. Fin 2004, la Suisse a remis au CAD un rapport rédigé sous la forme d'un mémorandum, dans lequel elle a dressé un bilan détaillé de ses activités. Au cours du premier trimestre 2005, des experts de Norvège, de Nouvelle-Zélande et du CAD procéderont à un examen approfondi de la coopération suisse au développement à la centrale ainsi que sur le terrain (programme en Bosnie et au Vietnam). Les résultats de «l'évaluation par les pairs» sont attendus pour l'automne 2005. Ils seront remis sous la forme d'un rapport contenant des observations et des recommandations à l'intention de la Suisse, qui se prononcera dès la publication du rapport ${ }^{3}$.

\section{Publication de lignes directrices et d'ouvrages de référence}

Le CAD adopte des lignes directrices et des ouvrages de référence destinés à aider les membres dans la conduite de leurs programmes de coopération. Parmi les récentes «Lignes directrices», citons le document Inscrire la coopération pour le développement dans une optique de prévention du terrorisme. Principaux points d'ancrage pour l'action, qui s'appuie sur les mesures prises par les donateurs face au terrorisme international (voir encadré ci-dessous).

\section{Inscrire la coopération pour le développement dans une optique de prévention du terrorisme. Principaux points d'ancrage pour l'action}

Partant du constat que "si le terrorisme international a des causes complexes, les motivations de ses acteurs et le choix de ses théâtres d'opération ne sont cependant pas sans lien avec les problèmes de développement", les gouvernements membres de I'OCDE représentés au sein du CAD ont engagé une réflexion sur les moyens les plus efficaces de soutenir l'effort mondial de lutte contre le terrorisme. Ces réflexions ont fait l'objet de la publication susmentionnée, dont les principales options ont été entérinées par le CAD lors de sa réunion de haut niveau de 2003.

Le document tente une évaluation des liens complexes entre les causes du terrorisme et le rôle que peuvent jouer les divers acteurs (pouvoirs publics, société civile, monde des affaires et, même, milieux du crime). II veut guider les initiatives prises par la communauté internationale et les gouvernements face au lien entre terrorisme et développement, et propose certains "points d'ancrage" dans les programmes de coopération au développement afin de mieux intégrer la lutte contre le terrorisme.

L'énoncé de cette politique, approuvée au niveau ministériel des pays de I'OCDE, a suscité de nombreuses réactions. Le Conseil canadien pour la coopération internationale (CCCI) a rédigé un document qui met sévèrement en garde les orientations du CAD en la matièrea. De même, des coalitions d'ONG ont fait cause commune pour présenter au CAD une déclaration conjointe qui remet en question la déclaration de principe du CAD, en invoquant un détournement de l'aide (voir ci-dessous l'encadré "The Reality of Aid 2004 ».

Sources: OCDE, Inscrire la coopération pour le développement dans une optique de prévention du terrorisme. Principaux points d'ancrage pour l'action, Paris, OCDE, 2003, disponible sur le site de I'OCDE, <Www.oecd.org/dataoecd/17/9/16175824.pdf>.

a Commentaire du CCCl relatif à la déclaration "Inscrire la coopération pour le développement dans une optique de prévention du terrorisme: principaux points d'ancrage pour l'action", octobre 2003, disponible sur le site <www.ccic.ca>.

3 DDC, Examen par les pairs du Comité d'aide au développement. La Suisse a encore du pain sur la planche, communiqué de presse, Berne, 11 octobre 2004. 


\section{Recommandations de l'OCDE}

Le CAD est habilité à adresser des recommandations à ses membres sur les questions de sa compétence. Parmi les exemples récents, citons la Recommandation de 2001 sur le déliement de l'aide aux pays les moins avancés.

$\mathbb{D}$ Annuaire 2003, $\mathrm{n}^{\circ}$ 1, chap. 2, «Coopération avec les pays en développement », le déliement de l'aide (pp. 15-16).

\section{Statistiques et rapports}

Le CAD assure la publication d'un rapport annuel sur les efforts et les politiques en matière de coopération au développement, qui fait référence dans le domaine. De plus, il fournit des données statistiques sur les apports d'aide publique au développement et d'autres ressources aux pays en développement et en transition, qui se fondent sur les notifications de ses membres ${ }^{4}$. La définition des composantes de l'APD fait régulièrement l'objet de discussions au sein du CAD. La «Liste des bénéficiaires de l'aide» est périodiquement revue par les membres du CAD.

Le rapport $2003^{5}$ insiste sur le rôle essentiel que doivent jouer les peuples et les gouvernements des pays concernés, soulignant que les politiques et les institutions des pays en développement restent les clés pour une amélioration durable des conditions de vie de leur population. Et rappelle que l'APD ne peut que faciliter le processus, et en particulier contribuer à en accélérer la progression. Le premier chapitre du rapport souligne le rôle des pays donateurs, d'une part en œuvrant pour une plus grande cohérence de leurs politiques en faveur du développement, d'autre part en accroissant le volume et l'efficacité de leur aide au développement. Dans le deuxième chapitre est présentée une analyse de l'évolution des apports d'aide aux pays en développement, et plus précisément des tendances observées au cours de la période récente dans le volume et la répartition de l'aide des membres du CAD. Enfin, le troisième chapitre fournit une évaluation des progrès qui restent à accomplir pour que soit assurée la réalisation des Objectifs du Millénaire pour le développement.

Annuaire 2003, $\mathrm{n}^{\circ}$ 1, chap. 2, «Coopération avec les pays en développement», le Comité d'aide au développement (pp. 13-18).

\subsubsection{Reality of Aid}

Reality of Aid $^{6}$ est une initiative non gouvernementale internationale «NordSud», qui rassemble plus de 40 réseaux de la société civile travaillant dans le domaine de la coopération internationale dans 22 pays de donateurs, mais également en Asie, en Amérique latine et en Afrique. Etabli en 1993, le projet a pour but de promouvoir les politiques nationales et internationales qui contribueront à la mise en place de stratégies de lutte contre la pauvreté.

\footnotetext{
Liens vers les statistiques en ligne de l'OCDE: <www.oecd.org/cad/stats/sdienligne>.

CAD (OCDE), Coopération pour le développement. Rapport annuel 2003, op. cit.

Le secrétariat de Reality of Aid est basé aux Philippines: c/o IBON Foundation, Inc. - 3/F, SCC Bldg., 4427 Int. Old Sta. Mesa - 1008 Manila, Philippines.
} 


\section{"The Reality of Aid 2004»}

Le rapport The Reality of Aid 2004 se concentre sur «la gouvernance et les droits humains" en faisant une critique cinglante de l'orientation actuelle de l'aide. Le rapport souligne " une tendance inquiétante chez les donateurs, qui invoquent l'amélioration de la gouvernance pour instaurer une gamme de plus en plus large de projets et programmes ayant peu à voir directement avec la réduction de la pauvreté" et indique que "les tentatives actuelles de détournement des fonds alloués à la réduction de la pauvreté en fonction des intérêts de sécurité des donateurs constituent l'expression la plus préoccupante du problème endémique de l'affectation des ressources réservées à l'aide internationale en fonction des priorités des pays riches »a. Pour illustrer cette affirmation, le rapport prend l'exemple de l'Australie, qui fait passer la réduction de la pauvreté après la sécurité dans la raison d'être de son aide internationale. De même, l'aide apportée par le Danemark dépend de la participation active des pays à la guerre contre le terrorisme. Quant au Japon, il a révisé sa Charte de l'aide au développement en ajoutant la "prévention du terrorisme». Pour la Communauté de travail des œuvres d'entraide, en charge du chapitre concernant la Suisse, le combat contre le terrorisme n'est pas un souci prioritaire de la politique étrangère helvétique et de sa politique de coopération au développement. La lutte contre le terrorisme est considérée comme une tâche urgente, mais commune à toutes les nations. Les auteurs relèvent également qu'une des préoccupations montantes au sein du Département des affaires étrangères concerne le risque, au nom de la lutte contre le terrorisme, de constater certaines violations des droits de l'homme ${ }^{\mathrm{b}}$.

Une déclaration signée par de nombreux membres du réseau Reality of Aid au sujet des préoccupations suscitées par la politique défendue par le CAD a souligné quatre impacts potentiels en lien avec «la guerre globale contre la terreur " et le développement:c

- politisation de l'aide: les relations entre donateurs et pays en voie de développement, dans le domaine de l'aide, vont être manipulées pour garantir l'appui politique à «la guerre contre la terreur" ;

\ nouveaux objectifs pour l'aide au développement: les objectifs de l'aide au développement vont être redéfinis pour inclure la lutte contre le terrorisme. L'objectif de lutte contre la pauvreté s'en trouve ainsi affaibli, car les budgets d'aide sont rarement augmentés pour tenir compte des nouveaux objectifs;

$\checkmark$ droits de l'homme: les droits de l'homme sont bafoués par de nouvelles législations antiterroristes. Et I'on observe une acceptation tacite de violations des droits de l'homme au nom de "la guerre contre la terreurn;

- crises oubliées et promesses non tenues: malgré la rhétorique sur la nécessité de s'en prendre aux racines du terrorisme, y compris la pauvreté et l'injustice, peu d'actions concrètes ont été menées. De plus, des engagements pris par les donateurs envers des pays directement touchés par «la guerre contre la terreur", à l'instar de l'Afghanistan, n'ont pas été tenus.

Source: Reality of Aid, The Reality of Aid 2004 : An Independant Review of Poverty Reduction and Developement Assistance, Manilla (Philippines); New York (USA), IBON Books; Zed Books, 2004.

a Conseil canadien pour la coopération internationale, L'aide internationale détournée au profit de la "guerre contre le terrorisme", communiqué de presse, 28 mai 2004.

b Reality of Aid, The Reality of Aid 2004 : An Independant Review of Poverty Reduction and Developement Assistance, op. cit., p. 295.

c British Overseas NGOs for Development (BOND), Global Security and Development (updated: July 2004), BOND Discussion Paper, <www.bond.org.uk/advocacy/globalsecurity.htm>. 


\section{Les activités de Reality of Aid}

\section{Rapports bisannuels}

Depuis 2002, le réseau publie un rapport thématique bisannuel évaluant l'efficacité de l'aide dans la perspective de la réduction de pauvreté, avec un accent particulier porté sur l'analyse qualitative du régime d'aide nationale et multilatérale. Les rapports Reality of Aid analysent et commentent les grandes tendances de la coopération internationale au développement et les performances des donateurs. L'originalité de la démarche procède de la provenance des sources, puisque les comptes-rendus nationaux sont confiés à des ONG du Nord et du Sud, partenaires du réseau. Fort de cet argument, Reality of Aid se présente comme un "correctif crédible des publications officielles sur l'aide au développement et l'éradication de la pauvreté »?

\section{Lobbying et dialogue politique}

Les rapports, largement distribués, favorisent un dialogue politique avec les fonctionnaires de ministères d'aide et les agences multilatérales (notamment au niveau du CAD), mais aussi avec des politiciens et des gouvernements, dans le but de rappeler aux donateurs leurs obligations en matière de lutte contre la pauvreté.

\section{«Advocacy»}

Par ses réseaux d'ONG, tant au Sud qu'au Nord, Reality of Aid tente de développer une vision et un agenda communs en matière de coopération au développement, qui puissent être partagés par tous les acteurs de la société civile participant au projet. Ces dernières années, avec l'inclusion systématique de réseaux du Sud, le projet a souligné l'importance de la mise en œuvre de stratégies régionales, spécifiques, appropriées et ciblées. Les rapports de Reality of Aid participent activement à l'identification d'une nouvelle approche de la coopération au développement.

\subsection{Volume de l'APD des pays du CAD et de la Suisse}

\subsubsection{APD fournie par l'ensemble des membres du Comité d'aide au développement (CAD)}

L'APD totale des pays membres du CAD a atteint 69 milliards de dollars en 2003, soit un montant sans précédent en valeur réelle comme en valeur nominale. Ce total représente $0,25 \%$ du revenu national brut (RNB) global des membres du CAD, contre $0,23 \%$ en 2002 et $0,22 \%$ en 2001 . La performance moyenne des pays du CAD se monte à $0,41 \%$. Cette augmentation de 2,3 milliards de dollars en termes réels en 2003 s'explique principalement par une croissance des dons bilatéraux en général (2 milliards de dollars), ainsi que par le début de l'aide à la reconstruction de l'Irak (comptabilisée à hauteur de 2 milliards de dollars pour 2003), mais, en contrepartie, on constate une baisse cyclique des contributions aux fonds concessionnels multilatéraux ( $-1,2$ milliard de dollars) et une petite diminution dans les prêts nets $(-500 \text { millions de dollars })^{8}$.

7 Site Internet: <www.realityofaid.org $>$.

8 CAD (OCDE), Augmentation modeste de l'aide au développement en 2003, communiqué de presse, 16 avril 2004. 
Tableau 1: APD de l'ensemble des pays du CAD, 1995-2003

\begin{tabular}{lccccccccc}
\hline APD nette du CAD & $\mathbf{1 9 9 5}$ & $\mathbf{1 9 9 6}$ & $\mathbf{1 9 9 7}$ & $\mathbf{1 9 9 8}$ & $\mathbf{1 9 9 9}$ & $\mathbf{2 0 0 0}$ & $\mathbf{2 0 0 1}$ & $\mathbf{2 0 0 2}$ & $\mathbf{2 0 0 3}$ \\
\hline Milliards de dollars & 58.9 & 55.4 & 48.3 & 51.9 & 56.4 & 53.7 & 52.3 & 58.2 & 69.0 \\
$\begin{array}{l}\text { Performance moyenne } \\
\text { des pays du CAD } \\
\text { (\% APD/RNB) }\end{array}$ & 0.41 & 0.39 & 0.38 & 0.39 & 0.39 & 0.39 & 0.39 & 0.41 & 0.41 \\
\hline APD des pays du CAD & & & & & & & & & \\
\hline \begin{tabular}{l} 
(\% du RNB) \\
\hline
\end{tabular} & 0,27 & 0,25 & 0,22 & 0,23 & 0,24 & 0,22 & 0,22 & 0,23 & 0,25 \\
\hline
\end{tabular}

Sources: CAD (OCDE), Coopération pour le développement. Rapport annuel 2003, op. cit., et Annexe statistique de la publication "Coopération pour le développement. Rapport 2004", disponible en ligne sur <www.oecd.org/document/10/ 0,2340,fr_2649_34447_34036298_1_1_1_1,00.html>.

Remarque: Les pays membres du CAD ont accru leur APD en faveur des pays en développement de 3,9\% en termes réels entre 2002 et 2003, après une hausse de $7.0 \%$ en termes réels entre 2001 et 2002. Les données exprimées en "valeur réelle" sont ajustées pour tenir compte de l'inflation et des fortes fluctuations des taux de change intervenues au cours des deux dernières années.

${ }^{a}$ Moyenne obtenue par l'addition des rapports APD/RNB pour chaque pays membre du CAD.

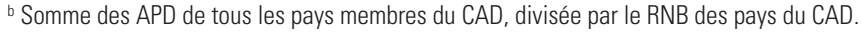

Tableau 2: APD nette par pays, 2002-2003 (chiffres de décembre 2004) (en millions de dollars et en pourcentage)

\begin{tabular}{|c|c|c|c|c|c|}
\hline & \multicolumn{2}{|c|}{2003} & \multicolumn{2}{|c|}{2002} & \multirow{2}{*}{ 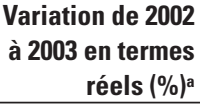 } \\
\hline & $\begin{array}{c}\text { APD } \\
\text { (mio. USD) }\end{array}$ & $\begin{array}{r}\text { APD/RNB } \\
(\%)\end{array}$ & $\begin{array}{c}\text { APD } \\
\text { (mio. USD) }\end{array}$ & $\begin{array}{r}\text { APD/RNB } \\
(\%)\end{array}$ & \\
\hline Etats-Unis & 16254 & 0.15 & 13290 & 0.13 & 20.4 \\
\hline Italie & 2433 & 0.17 & 2332 & 0.20 & -15.3 \\
\hline Autriche & 505 & 0.20 & 520 & 0.26 & -20.5 \\
\hline Japon & 8880 & 0.20 & 9283 & 0.23 & -9.2 \\
\hline Grèce & 362 & 0.21 & 276 & 0.21 & 5.7 \\
\hline Portugal & 320 & 0.22 & 323 & 0.27 & -19.4 \\
\hline Espagne & 1961 & 0.23 & 1712 & 0.26 & -7.8 \\
\hline Nouvelle-Zélande & 165 & 0.23 & 122 & 0.22 & 6.9 \\
\hline Canada & 2031 & 0.24 & 2004 & 0.28 & -12.7 \\
\hline Australie & 1219 & 0.25 & 989 & 0.26 & 0.4 \\
\hline Allemagne & 6784 & 0.28 & 5324 & 0.27 & 5.3 \\
\hline Royaume-Uni & 6282 & 0.34 & 4924 & 0.31 & 14.0 \\
\hline Finlande & 558 & 0.35 & 462 & 0.35 & 0.3 \\
\hline Irlande & 504 & 0.39 & 398 & 0.40 & 3.8 \\
\hline Suisse & 1299 & 0.39 & 939 & 0.32 & 19.7 \\
\hline France & 7253 & 0.41 & 5486 & 0.38 & 8.7 \\
\hline Belgique & 1853 & 0.60 & 1072 & 0.43 & 40.7 \\
\hline Suède & 2400 & 0.79 & 2012 & 0.84 & -2.8 \\
\hline Pays-Bas & 3981 & 0.80 & 3338 & 0.81 & -3.2 \\
\hline Luxembourg & 194 & 0.81 & 147 & 0.77 & 8.4 \\
\hline Danemark & 1748 & 0.84 & 1643 & 0.96 & -12.8 \\
\hline Norvège & 2042 & 0.92 & 1696 & 0.89 & 4.6 \\
\hline Total du CAD & 69029 & 0.25 & 58292 & 0.23 & 4.8 \\
\hline \multicolumn{6}{|l|}{ Pour mémoire: } \\
\hline Communauté européenne & 7173 & & 5448 & & 7.7 \\
\hline Pays membres de l'UE & 37139 & 0.35 & 29969 & 0.35 & 3.0 \\
\hline Pays du G-7 & 49917 & 0.21 & 42644 & 0.20 & 6.3 \\
\hline Pays non membres du G-7 & 19112 & 0.46 & 15648 & 0.47 & 0.5 \\
\hline
\end{tabular}

Source: CAD (OCDE), Annexe statistique de la publication "Coopération pour le développement. Rapport 2004", op. cit.

a Compte tenu de l'inflation et des fluctuations des taux de change. 
Les Etats-Unis demeurent le premier donateur d'aide au monde en volume (mais ils occupent le dernier rang en termes de rapport APD/RNB), suivis du Japon, de la France, de l'Allemagne et du Royaume-Uni. Seuls cinq pays atteignent l'objectif de $0,7 \%$ du RNB préconisé par les Nations unies pour l'APD: la Norvège $(0,92 \%)$, le Danemark $(0,84 \%)$, le Luxembourg $(0,81 \%)$, les PaysBas $(0,8 \%)$ et la Suède $(0,79 \%)$. Trois autres pays se sont fixé une date précise pour atteindre cet objectif: la Belgique en 2010, l'Irlande en 2007, et la France en 2012, en passant par un objectif intermédiaire de 0,5\% en 2007.

L'APD du Japon a pour sa part fléchi de 9,2\% en termes réels, pour s'établir à 8,9 milliards de dollars, soit $0,20 \%$ du RNB. Si les versements bruts sont restés relativement stables, les remboursements effectués au titre des prêts d'APD ont augmenté de manière sensible.

L'APD des Etats membres de l'UE s'est accrue de 2,2\% en termes réels en 2003, représentant $0,35 \%$ de leur RNB collectif. Déjà avant la Conférence de Monterrey, les membres de l'UE s'étaient engagés à porter leur APD globale à $0,39 \%$ de leur RNB pour 2006, et à titre individuel à un minimum de $0,33 \%$.

\subsubsection{Rapport d'Oxfam International "Le prix à payer "}

Oxfam International a rendu public, en décembre 2004, un nouveau rapport, Le prix à payer. Pourquoi les riches doivent investir maintenant dans la pauvreté, qui tempère l'optimisme des chiffres énoncés par le CAD (voir ci-dessus). Le rapport rappelle que les avancées visant à la réalisation des Objectifs du Millénaire pour le développement sont très éloignées des promesses faites en 2000. Et le rapport de constater que «les montants que les pays riches investissent dans la réduction de la pauvreté à l'échelle mondiale sont ridiculement petits. [...] Aujourd'hui, les pays riches donnent deux fois moins que dans les années 1960 en termes de pourcentage de leurs revenus. Entre 1960 et 1965, les pays riches dépensaient 0,48\% de leurs revenus nationaux en aide. Entre 1980 et 1985, ils ne dépensaient que $0,34 \%$. En 2003, cette moyenne avait chuté à $0,24 \%$ » .

\subsubsection{Aide publique et privée au développement fournie par la Suisse}

La Loi fédérale sur la coopération au développement et l'aide humanitaire internationales (1976) stipule que les deux offices fédéraux en charge de la mise en œuvre de l'aide au développement de la Confédération sont la Direction du développement et de la coopération (DDC) et le Secrétariat d'Etat à l'économie (seco). Les contributions des deux offices (y compris les annulations de dette comptabilisées dès 2003) constituent $90 \%$ de l'aide publique au développement (en faveur des pays en développement) et de l'aide publique (en faveur des pays en transition $)^{10}$ de la Suisse. Mais, comme le montrent les tableaux 3 et 4, un

9 Oxfam International, Le prix à payer. Pourquoi les riches doivent investir maintenant dans la pauvreté (résumé du rapport), décembre 2004, <www.oxfam.org/fr/pdf/Paying\%20the\%20Price\%20Summary \%20French.pdf>.

10 L'aide publique au développement (APD) est définie par l'OCDE comme l'ensemble des flux financiers qui proviennent d'organismes publics (Confédération, cantons et communes), accordés à des conditions de faveur (dons ou prêts à taux bonifiés) et qui ont pour but essentiel le développement économique et social des pays destinataires. Ils sont destinés à des pays ou territoires figurant dans la liste établie par le CAD (OCDE). Cette liste comprend 150 pays et territoires classés sous l'appella- 
certain nombre de contributions proviennent d'autres offices et départements fédéraux, qui sont également comptabilisées au titre de l'APD ou de l'AP. A cela s'ajoutent les contributions communales et cantonales, dont les contributions financières constituent $2,4 \%$ de l'APD bilatérale et 3\% de l'AP bilatérale. Ces montants sont versés en grande partie à des ONG suisses, soit directement, soit en passant par exemple par une fédération cantonale de coopération.

L'aide privée de la Suisse provient de dons du secteur privé ou de la population en faveur des institutions privées de développement (regroupées ici sous le nom d'ONG). Il s'agit de fonds alloués dans un but non lucratif par des fondations, des associations, des œuvres d'entraide ou d'autres organisations pour des actions de coopération au développement ou d'aide humanitaire à des partenaires dans les pays en développement ou en transition. Certaines ONG bénéficient de contributions du secteur public (Confédération, cantons, communes) pour financer des programmes de coopération au développement, mais ces montants ne sont pas pris en compte dans le chiffre figurant dans les tableaux 3 et 4 sous «aide privée »; ils sont comptabilisés dans l'aide publique.

En 2003, l'aide publique au développement (APD, voir tableau 3) de la Suisse a atteint 1748 millions de francs, soit 0,39\% du RNB. Par rapport à 2002 (1462 millions de francs), elle a subi une forte augmentation en chiffres absolus. L'aide bilatérale a atteint 1271 millions de francs (1192 millions en 2002) et l'APD multilatérale 477 millions (270 millions en 2002). L'augmentation enregistrée en 2003 s'explique par une notification différée des premières contributions suisses respectivement à l'AID-13 (13 ${ }^{\mathrm{e}}$ reconstitution du fonds de l'AID) et au FAfD-9 ( $9^{\mathrm{e}}$ reconstitution du FAfD), initialement prévues pour 2002, de même que par des ajustements statistiques ${ }^{11}$. L'aide privée a sensiblement augmenté, passant de 298 millions de francs en 2002 à 377 millions de francs en 2003.

L'aide publique de la Suisse aux pays en transition (voir tableau 4) est stable; elle s'est élevée à 104 millions de francs en 2003. La DDC et le seco collaborent pour définir des projets dans le domaine de la coopération technique et financière avec les pays d'Europe de l'Est et de la CEI. A noter que les ONG suisses travaillent également en faveur des pays en transition et ont récolté, en 2003, environ 15 millions de francs.

Annuaire 2005, $\mathrm{n}^{\circ}$ 1, chap. 3, «Coopération avec les pays d'Europe de l'Est et de la CEI».

tion «en développement», dont, en Europe, tous les pays de l'ex-Yougoslavie, l'Albanie, la Moldova et Malte. L'aide publique (AP) répond aux quatre mêmes critères que l'APD, mais elle est destinée aux pays dits en transition, soit 12 pays d'Europe centrale et orientale et 24 pays en développement plus avancés. Source: DDC/seco, Rapport annuel de la coopération internationale de la Suisse 2003, Berne, 2004, <www.deza.admin.ch/ressources/deza_product_fr_996.pdf>. La liste des pays bénéficiaires de l'aide (AP/APD) telle qu'établie par le CAD figure à la fin du présent Annuaire.

11 Voir la remarque au bas du tableau 3. 
Tableau 3: Aide publique de la Suisse aux pays en développement, 2003 (en millions de francs)

\begin{tabular}{|c|c|c|c|c|}
\hline & \multicolumn{3}{|c|}{2003} & \multirow{2}{*}{$\begin{array}{c}2002 \\
\text { Total } \\
\text { APD }\end{array}$} \\
\hline & $\begin{array}{c}\text { APD } \\
\text { bilatérale }\end{array}$ & $\begin{array}{c}\text { APD } \\
\text { multilatérale }\end{array}$ & $\begin{array}{l}\text { Total } \\
\text { APD }\end{array}$ & \\
\hline $\begin{array}{l}\text { Direction du développement } \\
\text { et de la coopération (DDC) }\end{array}$ & 865.0 & 407.2 & 1272.2 & 1049.8 \\
\hline Aide humanitaire & 234.7 & 28.5 & 263.2 & 267.5 \\
\hline Coopération au développement & 497.1 & 378.7 & 875.8 & 654.5 \\
\hline Coopération avec l'Est et la CEl & 70.4 & 0.0 & 70.4 & 66.6 \\
\hline $\begin{array}{l}\text { Contributions des programmes } \\
\text { aux ONG suisses }\end{array}$ & 62.7 & 0.0 & 62.7 & 61.1 \\
\hline Secrétariat d'Etat à l'économie (seco) & 240.2 & 12.0 & 252.2 & 231.0 \\
\hline Coopération au développement & 148.0 & 12.0 & 160.0 & 156.9 \\
\hline Coopération avec l’Est et la CEI & 52.2 & 0.0 & 52.2 & 74.1 \\
\hline Réaménagement des dettes & 40.0 & 0.0 & 40.0 & 0.0 \\
\hline \multicolumn{5}{|l|}{ Office fédéral des réfugiés } \\
\hline $\begin{array}{l}\text { Assistance aux réfugiés, } \\
\text { part comptabilisée dans I'APD }\end{array}$ & 48.6 & 0.0 & 48.6 & 60.4 \\
\hline $\begin{array}{l}\text { Département fédéral } \\
\text { des affaires étrangères }\end{array}$ & 31.7 & 12.7 & 44.4 & 34.9 \\
\hline $\begin{array}{l}\text { Projets dans le domaine de la } \\
\text { gestion civile des conflits }\end{array}$ & 31.1 & 0.0 & 31.1 & 23.0 \\
\hline Contributions multilatérales et autres actions & 0.6 & 12.7 & 13.3 & 11.9 \\
\hline Administration fédérale des finances & 0.0 & 14.7 & 14.7 & 21.0 \\
\hline Aide aux pays affectés par la crise du Golfe & 0.0 & 0.0 & 0.0 & 5.1 \\
\hline Contribution au FMI & 0.0 & 14.7 & 14.7 & 15.9 \\
\hline \multicolumn{5}{|l|}{$\begin{array}{l}\text { Office fédéral de l'environnement, } \\
\text { des forêts et du paysage }\end{array}$} \\
\hline Contribution au FEM et autres organisations & 4.5 & 22.5 & 27.0 & 19.3 \\
\hline \multicolumn{5}{|l|}{$\begin{array}{l}\text { Département fédéral de la défense, de la } \\
\text { protection de la population et des sports }\end{array}$} \\
\hline Actions de secours aux réfugiés du Kosovo & 41.4 & 0.0 & 41.4 & 0.5 \\
\hline Office fédéral de la santé publique & 0.2 & 4.9 & 5.1 & 7.4 \\
\hline \multicolumn{5}{|l|}{ Office fédéral de l'agriculture } \\
\hline Contributions à la FAO & 0.6 & 2.8 & 3.4 & 3.9 \\
\hline \multicolumn{5}{|l|}{ Office fédéral de l'éducation et de la science } \\
\hline Bourses à des étudiants étrangers en Suisse & 4.6 & 0.0 & 4.6 & 4.2 \\
\hline Confédération - divers & 3.7 & 0.2 & 3.9 & 4.7 \\
\hline Cantons et communes & 30.1 & 0.0 & 30.1 & 25.0 \\
\hline $\begin{array}{l}\text { Total de I'aide publique } \\
\text { au développement }\end{array}$ & 1270.6 & 477.1 & 1747.7 & 1462.1 \\
\hline Aide privée (ONG) & 376.9 & 0.0 & 376.9 & 298.2 \\
\hline
\end{tabular}

Source: DDC, Aide de la Suisse aux pays en développement et aux pays en transition. Statistiques 2003, Berne, à paraître en mars 2005.

Remarque: Les directives de I'OCDE relatives au calcul de I'APD sont régulièrement revues et adaptées à l'évolution de la coopération internationale. Aussi les prestations de la Suisse entrant en considération pour l'APD incluent-elles désormais davantage d'activités de promotion de la paix et dans le domaine de la sécurité, ainsi que les annulations de dette en faveur des pays en développement négociées dans le cadre du Club de Paris. Cela explique l'introduction dans les statistiques d'APD de la Suisse d'une nouvelle catégorie statistique ("Réaménagement des dettes»), ainsi que la prise en compte de contributions du DDPS en ce qui concerne les actions de maintien de la paix et de la sécurité. 
Tableau 4: Aide publique de la Suisse aux pays en transition, 2003 (en millions de francs)

\begin{tabular}{|c|c|c|c|c|}
\hline & \multicolumn{3}{|c|}{2003} & \multirow{2}{*}{$\begin{array}{r}2002 \\
\text { Total } \\
\text { AP } \\
\end{array}$} \\
\hline & $\begin{array}{c}\text { AP } \\
\text { bilatérale }\end{array}$ & $\begin{array}{c}\text { AP } \\
\text { multilatérale }\end{array}$ & $\begin{array}{r}\text { Total } \\
\text { AP } \\
\end{array}$ & \\
\hline $\begin{array}{l}\text { Direction du développement } \\
\text { et de la coopération (DDC) }\end{array}$ & 42.3 & 0.0 & 42.3 & 65.4 \\
\hline Aide humanitaire & 9.5 & 0.0 & 9.5 & 32.8 \\
\hline Coopération au développement & 0.8 & 0.0 & 0.8 & 0.6 \\
\hline Coopération avec l'Est et la CEI & 31.7 & 0.0 & 31.7 & 31.9 \\
\hline Contributions des programmes aux ONG suisse & 0.2 & 0.0 & 0.2 & 0.1 \\
\hline Secrétariat d’Etat à l'économie (seco) & 42.1 & 9.6 & 51.7 & 28.2 \\
\hline Coopération au développement & 0.0 & 0.0 & 0.0 & 0.2 \\
\hline Coopération avec l’Est et la CEI & 42.1 & 9.6 & 51.7 & 28.0 \\
\hline \multicolumn{5}{|c|}{ Département fédéral des affaires étrangères } \\
\hline Action en faveur du maintien de la paix & 0.3 & 0.0 & 0.3 & 0.2 \\
\hline \multicolumn{5}{|l|}{$\begin{array}{l}\text { Office fédéral de l'environnement, } \\
\text { des forêts et du paysage }\end{array}$} \\
\hline Contribution au FEM et autres organisations & 0.04 & 5.3 & 5.3 & 3.5 \\
\hline \multicolumn{5}{|c|}{ Office fédéral de l'éducation et de la science } \\
\hline Bourses à des étudiants étrangers en Suisse & 1.5 & 0.0 & 1.5 & 1.7 \\
\hline Cantons et communes & 2.7 & 0.0 & 2.7 & 3.0 \\
\hline Total de l'aide publique & 88.9 & 14.9 & 103.8 & 102.0 \\
\hline Aide privée (ONG) & 15.4 & 0.0 & 15.4 & 15.7 \\
\hline
\end{tabular}

Source: DDC, Aide de la Suisse aux pays en développement et aux pays en transition. Statistiques 2003, op. cit.

\subsection{Coopération au développement de la Suisse}

La DDC et le seco ont défini leurs objectifs et leurs stratégies dans des documents stratégiques distincts ${ }^{12}$. Dans les deux cas, l'objectif affiché est la lutte contre la pauvreté et l'amélioration des conditions de vie des personnes les plus défavorisées des pays bénéficiaires de l'aide apportée par la Suisse. La DDC mise avant tout sur la résolution des conflits, le développement social, la bonne gouvernance, l'encouragement des structures économiques et la préservation des ressources naturelles. Pour sa part, le seco définit les mesures économiques et commerciales de la coopération au développement.

En 2003 et 2004, deux nouveaux crédits-cadre relatifs à la coopération suisse au développement sont entrés en vigueur après leur adoption par les Chambres fédérales. Le sixième crédit-cadre concernant le financement des mesures de politique économique et commerciale est doté d'un montant de 970 millions, pour une durée de cinq ans au moins (2003-2008). Ces mesures économiques et commerciales représentent environ $15 \%$ de l'APD et leur mise en œuvre est confiée au $\operatorname{seco}^{13}$. Le $10^{\mathrm{e}}$ crédit pour «la continuation de la coopération technique et de l'aide financière en faveur des pays en développement» est entré en vigueur en 2004 pour une période minimale de quatre ans (2004-2007); d'un montant de 4,2 milliards de francs, ces fonds sont gérés par la $\mathrm{DDC}^{14}$.

12 seco, Stratégie 2006, Berne, 2002; DDC, Stratégie 2010, Berne, 2001.

13 Conseil fédéral, Message concernant la continuation du financement des mesures de politique économique et commerciale au titre de la coopération au développement du 20 novembre 2002 (FF 2003 $155)$.

14 Conseil fédéral, Message concernant la continuation de la coopération technique et de l'aide financière en faveur des pays en développement (2004-2007) du 28 mai 2003 (FF 2003 4155). 
Da Annuaire 2004, $\mathrm{n}^{\circ}$ 1, chap. 2, «Coopération avec les pays en développement», présentation des deux messages (DDC et seco) adoptés en 2003 (pp. 13-39).

\subsubsection{Conférence annuelle de la coopération au développement}

La Conférence annuelle de la coopération au développement, organisée par la DDC et le seco, s'est tenue en août 2004 à Saint-Gall et a porté sur les nombreuses perspectives de la collaboration entre la Suisse et l'Afrique du Sud; 1200 invités ont pris part à la manifestation, à laquelle ont également participé le professeur Kader Asmal, représentant du gouvernement sud-africain, la conseillère fédérale Micheline Calmy-Rey, ainsi que les directeurs respectifs de la DDC et du seco, Walter Fust et Jean-Daniel Gerber ${ }^{15}$.

Cette conférence a été l'occasion de présenter la réorganisation de la coopération suisse en Afrique du Sud. Depuis les premières élections libres d'avril 1994, la DDC a un bureau à Johannesburg et mène un «programme spécial» pour l'Afrique du Sud, dont le but était de contribuer à réaliser la transition dans «l'après-apartheid». Avec le programme de promotion de la paix du DFAE et le soutien du seco au développement de petites et moyennes entreprises, l'ensemble de la coopération suisse s'est élevé à 14 millions en 2003 et devrait atteindre 15,2 millions en 2004. Fin 2004 et après dix ans d'existence, le programme de la DDC sera converti en programme régional pour l'Afrique méridionale, avec comme priorité la gouvernance, la lutte contre le VIH/sida et la gestion des ressources naturelles ${ }^{16}$.

\subsubsection{5 - Année internationale du microcrédit}

L'Année internationale du microcrédit ${ }^{17}$ a été ouverte en novembre 2004 dans des Bourses du monde entier. La Suisse a déjà beaucoup développé le secteur de la microfinance, sous l'impulsion de la DDC et du seco, et grâce à l'engagement d'œuvres d'entraide et de fondations.

En Suisse, le lancement de cette année internationale a été marqué par la collaboration entre le seco et une initiative privée, «responsAbility ${ }^{18}$, plate-forme d'investissements à caractère social soutenue par des acteurs privés (groupe Raiffeisen, Baumann \& C ${ }^{\mathrm{ie}}$, Credit Suisse, Alternative Bank ABS et la Swiss Re) et axée spécifiquement sur les pays en développement. «ResponsAbility» a pour vocation de servir de relais entre le capital privé et institutionnel et les initiatives prometteuses dans les pays en développement. Son objectif est de permettre la génération de revenus à travers les activités des microentreprises. La plate-forme a reçu un capital de départ de 3 millions de dollars de la part du seco.

DDC/seco, Conférence annuelle de la coopération au développement 2004 à Saint-Gall. Rétrospective: les dix ans de la nouvelle Afrique du Sud - un partenariat pour les jeunes, août 2004.

16 «Dix ans après la fin officielle de l'apartheid, la Suisse tourne la page et réorganise son aide à l'Afrique du Sud», Le Temps, 10 juillet 2004; «Südafrika-Hilfe unter neuem Vorzeichen», Neue Zürcher Zeitung, 26. Juli 2004.

17 Site officiel de l'Année internationale du microcrédit : <www.yearofmicrocredit.org >.

$18<$ www.responsAbility.ch>. 


\section{La microfinance}

La microfinance comprend des services bancaires destinés à des personnes pauvres mais actives économiquement, dans les pays en développement: il ne s'agit pas de donner une aumône mais d'aider ces personnes à accroître leur autonomie par leur motivation à créer une entreprise. L'accès à des crédits souvent situés dans une fourchette de 50 à 100 francs suisses permet fréquemment à ces petites entreprises ou microentreprises d'accroître considérablement leur chiffre d'affaires, leur rentabilité et donc leur revenu. La microfinance, conçue comme une aide à l'autoassistance, s'est avérée un moyen efficace de lutter contre la pauvreté, le taux de remboursement étant près de $100 \%$. Toutefois, on estime que moins de $10 \%$ des quelque 500 millions de petites entreprises et microentreprises recensées actuellement ont accès à des services financiers. II existe donc là un énorme potentiel de développement, qui peut être exploité durablement avec des fonds uniquement privés.

Source: seco, Année internationale du microcrédit, communiqué de presse, 18 novembre 2004.

Tableau 5: Répartition géographique de I'APD bilatérale de la DDC au titre de la coopération au développement, 2003 (en millions de francs)

\begin{tabular}{|c|c|c|c|}
\hline$\overline{\text { Afrique }}$ & 161.1 & Amérique latine (suite) & \\
\hline Pays à faible revenu & 128.6 & Brésil & 2.0 \\
\hline Tanzanie & 22.4 & Guatemala & 2.0 \\
\hline Burkina Faso & 18.5 & El Salvador & 1.9 \\
\hline Mozambique & 16.6 & Rép. dominicaine & 1.0 \\
\hline Tchad & 11.6 & Autres pays & 2.6 \\
\hline Mali & 10.6 & Amérique du Sud non ventilé & 2.1 \\
\hline Niger & 9.9 & Amérique N. et $\mathrm{C}$. non ventilé & 9.1 \\
\hline Bénin & 9.2 & Amérique non spécifié & 3.0 \\
\hline Autres pays & 6.4 & Asie & 148.8 \\
\hline Rwanda & 5.7 & Pays à faible revenu & 120.4 \\
\hline Madagascar & 4.5 & Inde & 30.2 \\
\hline Cap-Vert & 3.4 & Népal & 18.4 \\
\hline Sénégal & 3.3 & Pakistan & 16.6 \\
\hline Cameroun & 2.9 & Bangladesh & 14.1 \\
\hline Ethiopie & 1.4 & Vietnam & 12.8 \\
\hline Guinée-Bissau & 1.0 & Bhoutan & 7.5 \\
\hline Burundi & 1.0 & Laos & 4.1 \\
\hline Pays à revenu intermédiaire & 13 & Corée (R. dém.) & 4.1 \\
\hline Afrique du Sud & 7.9 & Afghanistan & 4.1 \\
\hline Tunisie & 1.5 & Cambodge & 3.7 \\
\hline Maroc & 1.2 & Indonésie & 3.0 \\
\hline Autres pays & 2.4 & Autres pays & 1.9 \\
\hline Nord du Sahara non ventilé & 0.1 & Pays à revenu intermédiaire & 19.0 \\
\hline Sud du Sahara non ventilé & 18.2 & Zones sous admin. palestinienne & 10.4 \\
\hline Afrique non spécifié & 1.2 & Chine & 2.9 \\
\hline Amérique latine & 86.1 & Sri Lanka & 1.8 \\
\hline Pays à faible revenu & 12.5 & Philippines & 1.5 \\
\hline Nicaragua & 8.9 & Autres pays & 2.4 \\
\hline Haïti & 3.5 & Moyen-Orient non ventilé & 0.6 \\
\hline Pays à revenu intermédiaire & 59.5 & Asie du Sud non ventilé & 0.4 \\
\hline Bolivie & 18 & Asie non spécifié & 8.3 \\
\hline Pérou & 14 & Europe & 3.1 \\
\hline Equateur & 9.4 & Pays à revenu intermédiaire & 2.8 \\
\hline Cuba & 3.3 & Turquie & 2.7 \\
\hline Colombie & 2.7 & Autres pays & 0.1 \\
\hline Honduras & 2.4 & Europe, non ventilé & 0.3 \\
\hline Non ventilé par continent & & & 160.7 \\
\hline APD bilatérale totale & & & 560 \\
\hline
\end{tabular}

Source: DDC, Service statistique, décembre 2004. 


\subsubsection{Répartition géographique de l'aide bilatérale}

En 2003, la DDC a versé environ 567 millions de francs d'APD au titre de sa coopération bilatérale au développement. Ce chiffre est relativement stable par rapport à 2002 (546 millions de francs). La coopération bilatérale de la DDC bénéficie en grande partie à l'Afrique (29\%) et à l'Asie (27\%), puis à l'Amérique latine $(15 \%)$ et à l'Europe $(1 \%)$, le reste étant destiné à des pays en développement sans spécification (29\%). A noter également que 47\% de l'APD bilatérale sont attribués à des pays à faible revenu et que $17 \%$ bénéficient aux pays à revenu intermédiaire; les $36 \%$ restant ne sont pas ventilés.

\subsubsection{Evaluation des lignes directrices de la DDC en matière de promotion des droits de l'homme}

La DDC a publié en mars 2004 les résultats d'une évaluation sur l'influence de ses lignes directrices dans le domaine de la promotion des droits humains ${ }^{19}$. L'évaluation analyse l'influence de deux documents publiés par la DDC sur le travail concret de l'aide (les lignes directrices de la DDC Promotion des droits de l'homme et coopération au développement publiées en 1997 et le document sur l'Etat de droit paru en 1998) ${ }^{20}$. Il s'agissait de voir dans quelle mesure ces documents ont servi de cadre de référence à la DDC et à ses partenaires et d'examiner leur influence sur le dialogue politique.

Voici quelques-unes des conclusions et recommandations de cette évaluation:

- la publication de ces lignes directrices était incontestablement opportune, pour mieux tenir compte du contexte et de l'importance des politiques de promotion des droits humains, suite au drame du Rwanda et pour le soutien aux pays en transition;

$\checkmark$ si la publication de ces lignes directrices était importante, il aurait fallu diffuser plus largement ces documents, notamment auprès des partenaires sur le terrain, et les utiliser davantage pour la formation du personnel de la DDC;

- les évaluateurs ont constaté que le contenu de ces rapports était finalement peu connu par les collaborateurs de la DDC;

$\checkmark$ les documents n'engageant que la DDC, ces lignes directrices n'ont qu'une influence très faible sur d'autres offices de l'administration fédérale, alors que la cohérence des politiques des différents offices devrait être renforcée;

$\checkmark$ les projets de la DDC spécifiquement créés pour promouvoir les droits de l'homme ont augmenté ces dernières années mais cela représente encore une part très faible du volume d'APD $(2,6 \%$ seulement du volume total des programmes bilatéraux de la DDC);

19 SDC, Evaluation 2004/1. SDC'S Human Rights and Rule of Law Guidance Documents: Influence, Effectiveness and Relevance within SDC, Bern, March 2004, 126 p.

20 La DDC a publié trois documents stratégiques dans le domaine de la promotion des droits humains: Promotion des droits de l'homme et coopération au développement, Berne, 1997; Le concept d'Etat de droit et ses implications pour la coopération au développement, Berne, 1998; L'action de la Suisse pour les peuples autochtones, Berne, 1999. Les documents sont disponibles sur <www.deza.ch>. 
- la publication des lignes directrices n'est pas suffisante pour changer réellement la politique; il faut aussi les faire connaître au personnel et les rendre visible dans les programmes concrets de coopération;

- les évaluateurs relèvent aussi les problèmes liés à la multiplication de documents publiés par la DDC sur des thèmes prioritaires. De nouveaux thèmes apparaissent et s'ajoutent aux autres thèmes prioritaires existants.

\subsection{Mesures de politique économique et commerciale du seco}

Par la mise en œuvre des mesures de politique économique et commerciale, le seco vise à favoriser des conditions-cadre propices à une croissance économique dans les pays du Sud et de l'Est. Il encourage l'intégration de ces pays à l'économie mondiale en conduisant des programmes de soutien macroéconomique ainsi que de promotion des investissements et du commerce. La lutte contre la pauvreté est l'objectif central poursuivi par le seco, comme en témoigne l'Agenda 2010. Pour la réduction de la pauvreté adopté en $2004^{21}$.

Tableau 6: Répartition géographique de I'APD/AP bilatérale du seco, 2003 (en millions de francs et en pourcentage)

\begin{tabular}{|c|c|c|c|c|c|c|c|}
\hline $\begin{array}{l}\text { Instruments pour } \\
\text { I'APD/AP }\end{array}$ & Afrique & $\begin{array}{l}\text { Amérique } \\
\text { latine }\end{array}$ & Asie & $\begin{array}{l}\text { Non spé- } \\
\text { cifié APD }\end{array}$ & $\begin{array}{l}\text { Europe } \\
(\text { AP) }\end{array}$ & Total & $\begin{array}{c}\text { Pourcen- } \\
\text { tage par } \\
\text { instrument }\end{array}$ \\
\hline $\begin{array}{l}\text { Mesures destinées à } \\
\text { favoriser l'engagement de } \\
\text { ressources du secteur privé }\end{array}$ & 14.1 & 11.7 & 19.4 & 7.8 & 24.4 & 77.4 & 26.8 \\
\hline Financements mixtes & 2.4 & 3.3 & 12.1 & 0.0 & 47.2 & 65.0 & 22.5 \\
\hline $\begin{array}{l}\text { Aide à la balance } \\
\text { des paiements }\end{array}$ & 24.9 & 9.0 & 0.3 & 7.7 & 2.8 & 44.7 & 15.4 \\
\hline Annulation de dettes & 40.0 & 0.0 & 0.0 & 0.0 & 0.0 & 40.0 & 13.8 \\
\hline Promotion commerciale & 2.4 & 2.5 & 2.2 & 12.0 & 7.6 & 26.7 & 9.2 \\
\hline Etudes et autres & 0.1 & 0.0 & 0.0 & 5.2 & 12.2 & 17.5 & 6.1 \\
\hline Mesures de désendettement & 7.5 & 0.0 & 0.0 & 2.3 & 0.0 & 9.8 & 3.4 \\
\hline Produits de base & 7.5 & 0.0 & 0.0 & 0.6 & 0.0 & 8.1 & 2.8 \\
\hline Total & 98.9 & 26.5 & 34.0 & 35.6 & 94.2 & 289.2 & 100 \\
\hline Pourcentage par continent & 34.2 & 9.2 & 11.7 & 12.3 & 32.6 & 100 & - \\
\hline
\end{tabular}

Source: DDC, Service statistique, décembre 2004.

a Sous la rubrique "Europe» sont comptabilisés les montants d'aide publique versés aux pays d'Europe centrale, d'Europe de l'Est et de la CEl, et d’Europe du Sud-Est, ainsi que des versements non spécifiés.

\subsubsection{Versements d'aide bilatérale du seco en 2003}

En 2003, le seco a versé plus de 289 millions de francs pour mettre en œuvre les mesures bilatérales de politique économique et commerciale. Le tableau 6 présente les versements bilatéraux du seco pour les pays en développement ${ }^{22}$ (aide

21 seco, «Les stratégies de lutte contre la pauvreté», thème du mois, La Vie économique (revue de politique économique du DFE), 08/2004.

22 En 2003, la coopération au développement a concentré son aide sur 16 pays prioritaires, dont la répartition est la suivante: en Afrique, Burkina Faso, Egypte, Ghana, Mozambique, Tanzanie, Maroc, République d'Afrique du Sud et Tunisie; en Amérique latine, Pérou, Amérique centrale et Bolivie; et en Asie, Chine, Inde, Jordanie, Vietnam et Indonésie. A noter également l'accent spécifique mis par le seco sur un partenariat avec les Etats méditerranéens. 
publique au développement APD) et pour les pays en transition (aide publique AP). Un peu plus du tiers des fonds versés par le seco au titre de l'aide bilatérale est attribué à des pays d'Afrique; l'Asie et l'Amérique latine reçoivent respectivement environ $10 \%$. Un deuxième tiers est attribué, au titre de l'aide publique, aux pays d'Europe centrale, d'Europe de l'Est et de la CEI, et d'Europe du Sud-Est. La tendance amorcée depuis l'utilisation du $5^{\mathrm{e}}$ crédit-cadre visant notamment à soutenir les nouveaux instruments du seco favorisant le secteur privé se confirme puisqu'elle absorbe environ un quart de l'aide bilatérale (27\%). Plus de $20 \%$ ont été attribués à des financements mixtes.

Annuaire 2005, $\mathrm{n}^{\circ}$ 3, sous-chap. 3.4, dépenses de la DDC et du seco en 2003 pour les pays d'Europe de l'Est et de la CEI.

\subsection{2. seco - Agenda 2010. Pour la réduction de la pauvreté}

La Stratégie 2006 constitue la base stratégique et opérationnelle du programme de coopération du seco; 1'Agenda 2010. Pour la réduction de la pauvretée $e^{23}$ est le résultat «d'une démarche participative et d'un processus d'apprentissage au sein du seco». Ce document donne de nouvelles impulsions à certains éléments clés de la Stratégie 2006 et précise la manière dont le seco entend consolider ses opérations afin de garantir sa contribution aux Objectifs du Millénaire pour le développement (OMD). Le document reflète la philosophie du seco, visant à «la promotion d'une économie de marché et d'une croissance économique durable ${ }^{24}$. Et le seco de se positionner clairement par rapport aux OMD: «Nous participons pleinement aux efforts concertés de la Suisse pour atteindre les OMD en aidant à traduire un engagement politique mondial en actions spécifiques en faveur du développement. Nous estimons cependant que les OMD ont un champ d'action relativement limité, avec un accent prononcé sur les aspects sociaux et environnementaux. Ces derniers sont indispensables et prioritaires pour sensibiliser le public, mais nous sommes convaincus que la lutte contre la pauvreté doit être appréhendées dans une perspective plus large, incluant également la promotion de la croissance économique et le développement du secteur privé, par le biais de l'investissement et du commerce. $»^{25}$

L'intérêt du document réside dans la clarification des objectifs poursuivis par le seco en matière de lutte contre la pauvreté dans un contexte de mondialisation économique, mais surtout le seco prend un certain nombre d'engagements pour améliorer l'efficacité de l'aide et en mesurer les résultats. L'Agenda 2010 identifie également des mesures spécifiques supplémentaires afin de renforcer la dimension de l'impact pro-poor de ses instruments et opérations.

\subsubsection{Participation du seco à l'Année internationale du riz (2004)}

L'ONU et la FAO ont déclaré 2004 Année internationale du riz ${ }^{26}$ afin de développer de nouvelles stratégies communes de lutte contre la faim et la pauvreté. A cette occasion, le seco a mené une campagne d'information, notamment sous

23 seco, Agenda 2010. Pour la réduction de la pauvreté, Berne, 2004, <www.seco-cooperation.ch/impe$\mathrm{ria} / \mathrm{md} /$ content/publikationen/35.pdf $>$.

24 Ibid., p. 8.

25 Ibid., p. 6.

26 Site officiel de l'Année internationale du riz $2004:$ : <www.fao.org/rice2004>. 
l'angle du commerce équitable en collaborant étroitement avec Claro Fair Trade $\mathrm{AG}^{27}$.

\subsection{La coopération multilatérale}

La DDC et le seco sont tous deux engagés dans la coopération multilatérale, tant financièrement que par leur participation aux travaux des principales enceintes. La coopération multilatérale de la DDC se concentre sur les activités des Nations unies (agences spécialisées et aide humanitaire) ${ }^{28}$. Le seco et la DDC sont responsables pour les relations et des activités de la Suisse avec le groupe de la Banque mondiale, avec les banques régionales de développement (Banques africaine, asiatique et interaméricaine de développement). Le seco est responsable pour les relations avec la Banque européenne pour la reconstruction et le développement. Le secteur du seco «Institutions financières multilatérales» définit, en étroite consultation entre le seco et la DDC, la stratégie et la position de la Suisse au sein de ses institutions partenaires (voir ci-dessous le point 2.6.2).

La complémentarité entre actions bilatérales et multilatérales a été récemment rappelée par Walter Fust dans un éditorial de la revue Un seul monde. Le directeur de la DDC y rappelle que «la complexité des thèmes et le volume des moyens nécessaires confèrent depuis quelque temps plus de poids au multilatéralisme de l'aide [...]. La politique est définie au sein d'organisations et de forums multilatéraux. Ensuite, elle est mise en œuvre par les institutions multilatérales et des partenaires bilatéraux. Il apparaît dès lors qu'aide bilatérale et aide multilatérale sont interdépendantes. Des institutions financières multilatérales ne peuvent par exemple investir leurs crédits à bon escient que lorsque leurs partenaires disposent des capacités nécessaires pour réaliser des projets prévus. Or le développement ou la promotion de ces capacités incombent souvent aux acteurs bilatéraux $»^{29}$.

\subsubsection{Versements multilatéraux de la Suisse en 2003}

L'aide multilatérale est une composante importante de la coopération avec les pays en développement ou en transition et représente un tiers de l'aide publique de la Suisse (477 millions de francs en 2003). L'engagement de la Suisse auprès des agences multilatérales s'organise autour de deux axes majeurs: le premier concerne les agences spécialisées des Nations unies, pour lesquelles la Suisse a participé à hauteur de 140 millions en 2003; le second concerne les institutions financières de développement (285 millions). Les contributions multilatérales de la Suisse sont stables entre 2002 et 2003, à l'exception de deux importantes contributions suisses à l'AID-13, d'un montant de 177 millions de francs, et au FAfD-9, de 72 millions de francs.

27 seco, Année internationale du riz 2004. Une action du seco en faveur du commerce équitable «Le riz, c'est la vie», Berne, avril 2004.

28 DDC, Stratégie multilatérale de la DDC, Berne, 2002.

29 DDC, «Coopération multilatérale: affronter ensemble les grands problèmes pour mieux aider les pauvres» (dossier), Un seul monde, n 4, décembre 2004. 
Tableau 7: APD multilatérale de la Suisse, contributions générales, 2003 (en millions francs)

\begin{tabular}{|c|c|c|}
\hline & 2003 & 2002 \\
\hline Organisations des Nations unies (NU) & 139.8 & 144.2 \\
\hline Programme des NU pour le développement (PNUD) & 52.0 & 52.0 \\
\hline Fonds des NU pour l'enfance (UNICEF) & 18.0 & 18.0 \\
\hline Haut-Commissariat pour les réfugiés (HCR) & 13.0 & 13.5 \\
\hline Fonds des NU pour la population (FNUAP) & 12.5 & 12.5 \\
\hline $\begin{array}{l}\text { Office de secours et de travaux des NU pour les réfugiés de Palestine } \\
\text { dans le Proche-Orient (UNRWA) }\end{array}$ & 10.8 & 11.8 \\
\hline Programmes spéciaux de l'Organisation mondiale de la santé (OMS) & 5.0 & 5.0 \\
\hline Programme commun des NU sur le VIH/sida (ONUSIDA) & 4.0 & 4.0 \\
\hline Bureau des NU de la coordination des affaires humanitaires (OCHA) & 2.2 & 2.0 \\
\hline Organisation des NU pour le développement industriel (ONUDI) & 1.8 & 1.9 \\
\hline Programme alimentaire mondial (PAM) & 1.5 & 1.5 \\
\hline Fonds des NU pour le développement de la femme (UNIFEM) & 0.9 & 0.8 \\
\hline Programme des Volontaires des NU (VNU) & 0.6 & 0.6 \\
\hline Convention des NU sur la lutte contre la désertification (CLD) & 0.3 & 0.6 \\
\hline Autres organisations des NU & 17.3 & 20.0 \\
\hline Institutions financières de développement & 285.2 & 86.8 \\
\hline Banque africaine de développement (BAfD) & 1.8 & 1.8 \\
\hline Fonds africain de développement (FAfD) & 71.6 & 46.0 \\
\hline Fonds asiatique de développement (FAsD) & 13.9 & 13.9 \\
\hline Banque interaméricaine de développement (BID) & 1.3 & 1.5 \\
\hline Association internationale de développement (AID) & 176.9 & - \\
\hline Fonds pour les pays pauvres très endettés (PPTE) & 4.9 & 7.6 \\
\hline Facilité du FMI pour la réduction de la pauvreté et la croissance (FRPC) & 8.7 & 9.5 \\
\hline Fonds fiduciaire du Fonds monétaire international (FMI) & 6.0 & 6.5 \\
\hline Autres institutions multilatérales & 52.1 & 38.9 \\
\hline Fonds pour l'environnement mondial (FEM) & 17.7 & 11.6 \\
\hline Groupe consultatif pour la recherche agricole internationale (CGIAR) & 10.8 & 10.9 \\
\hline Organisation internationale des bois tropicaux (OIBT) & 4.6 & 4.4 \\
\hline Agence intergouvernementale de la francophonie (AIF) & 4.3 & 4.4 \\
\hline Protocole de Montréal relatif à des substances qui appauvrissent la couche d'ozone & 4.0 & 3.0 \\
\hline Centre international de recherche sur les maladies diarrhéiques (ICDDR) & 1.1 & 0.9 \\
\hline Organisation internationale des migrations (OIM) & 1.0 & 1.1 \\
\hline Union internationale pour la conservation de la nature (UICN) & 0.7 & 1.8 \\
\hline Autres institutions multilatérales ${ }^{\mathrm{a}}$ & 7.9 & 1.1 \\
\hline Total de I'APD multilatérale & 477.1 & 270.0 \\
\hline
\end{tabular}

Source: DDC, Service statistique, décembre 2004.

a D'après les directives du CAD, les contributions au Fonds mondial de lutte contre le sida, la tuberculose et le paludisme (GF-ATM) sont comptabilisées comme versements à une organisation multilatérale à partir de 2003.

\subsubsection{Stratégie de la Suisse en matière d'aide au développement multilatérale et lignes directrices d'action pour la coopération avec la Banque mondiale}

Le Comité interdépartemental pour le développement et la coopération internationaux (CIDCI) a adopté en 2004 la Stratégie de la Suisse en matière de coopération multilatérale au développement et a décidé que des principes stratégiques sont fixés pour chaque organisation par le biais de lignes d'action institutionnelles.

La Stratégie de la Suisse en matière d'aide au développement multilatérale ${ }^{30}$ vise à renforcer la position de la Suisse dans le système multilatéral et à mieux

30 Document à paraître. 
utiliser les synergies entre la coopération multilatérale et l'aide directe au développement. Le document précise les objectifs poursuivis par la Suisse, à savoir la consolidation de l'architecture internationale de développement, une coopération axée sur les résultats et le choix stratégique de partenaires multilatéraux. Il est rappelé que les intérêts des pays partenaires mais aussi de la société civile suisse, du secteur privé et des milieux scientifiques doivent également être pris en compte. Ce document sert de cadre directeur à moyen terme pour la coopération avec les principaux acteurs multilatéraux de l'aide au développement.

Les Lignes d'action institutionnelle pour la coopération de la Suisse avec la Banque mondiale ${ }^{31}$ servent, d'une part, à régir le travail quotidien du seco et de la DDC, chargés de la mise en œuvre conjointe de la stratégie suisse, et, d'autre part, à informer le public intéressé sur les activités de la Suisse au sein des institutions multilatérales. La brochure souligne que la coopération avec le groupe de la Banque mondiale est l'un des principaux moyens d'influence de la Suisse dans la coopération internationale. Il est rappelé que l'engagement financier auprès de la Banque mondiale fait de la Suisse un acteur important dans le dialogue international, dont le secteur privé suisse profite, puisque l'industrie d'exportation suisse peut participer aux marchés publics de la BM. Depuis sa création, l'économie suisse a fourni au groupe de la BM des biens et des services pour un montant total de 6 milliards de francs, soit 3,5\% des adjudications de la Banque ${ }^{32}$.

\subsection{ONG suisses:}

stratégie de la Communauté de travail des œuvres d'entraide

De 2002 à 2004, la Communauté de travail (CT) a mené, avec les responsables de ses six organisations membres (Swissaid, Action de Carême, Pain pour le prochain, Helvetas, Caritas et l'EPER), un large débat sur ses orientations futures et le contenu de ses principaux axes stratégiques. Deux documents de fond ont résulté de ce processus et ont été publiés en automne 2004. Le premier, Lignes directrices de politique de développement, constitue le programme politique de l'organisation; il est publié, conjointement avec une analyse du contexte international faite par le directeur de la CT, Peter Niggli, dans le livre La mondialisation, et après... Quel développement au XXI ${ }^{e}$ siècle ${ }^{33}$. Le deuxième document, Stratégie de la Communauté de travail, précise le mandat, les champs d'action, le mode de travail ainsi que les publics cibles ${ }^{34}$.

$\mathbb{D}$ Annuaire 2004, $\mathrm{n}^{\circ} 2$, dossier Les $O N G$ de développement: rôles et perspectives (vol. $23, \mathrm{n}^{\circ} 2$, décembre 2004). Le dossier présente notamment de manière détaillée la structure du financement des ONG suisses, la répartition géographique de l'aide des ONG et la politique de la DDC à leur égard.

31 seco/DEZA, Die Schweiz und die Weltbank. Leitlinie, Bern, 2004, <www.deza.ch/ressources/deza_ product_de_1240.pdf $>$.

32 seco/DDC, Bulletin d'information. Coopération avec la Banque mondiale et les institutions financières régionales, Berne, novembre 2004.

33 Peter Niggli, La mondialisation, et après... Quel développement au XXI siècle ?, Lausanne, Communauté de travail Swissaid - Action de Carême - Pain pour le prochain - Helvetas - Caritas - EPER, 2004.

34 CT, «La stratégie de la Communauté de travail », Dossiers, n 3, octobre 2004. 


\section{SOURCES}

CAD (OCDE), Coopération pour le développement. Rapport annuel 2003, Les dossiers du CAD, vol. 5 , $\mathrm{n}^{\circ}$ 1, Paris, OCDE, 2004,

OCDE, communiqués de presse parus en 2004.

OCDE, Inscrire la coopération pour le développement dans une optique de prévention du terrorisme. Principaux points d'ancrage pour l'action, Paris, OCDE, 2003.

Conseil fédéral, Message concernant la continuation de la coopération technique et de l'aide financière en faveur des pays en développement (2004-2007) du 28 mai 2003 (FF 2003 4155).

Conseil fédéral, Message concernant la continuation du financement des mesures de politique économique et commerciale au titre de la coopération au développement du 20 novembre 2002 (FF 2003 $155)$.

DDC, communiqués de presse parus en 2004.

DDC, Créer des perspectives pour une existence digne. Principes de la DDC en matière de lutte contre la pauvreté, Berne, 2004.

DDC, Message concernant la continuation de la coopération technique et de l'aide financière en faveur des pays en développement (2004-2007), version abrégée, Berne, mai 2003.

DDC, Newsletter, parutions en 2004.

DDC, Stratégie multilatérale de la DDC, Berne, 2002.

DDC, Un seul monde, parutions en 2004. Notamment «Coopération multilatérale: affronter ensemble les grands problèmes pour mieux aider les pauvres » (dossier), n 4 , décembre 2004.

DDC/seco, Conférence annuelle de la coopération au développement 2004 à Saint-Gall. Rétrospective: les dix ans de la nouvelle Afrique du Sud - un partenariat pour les jeunes, août 2004.

DDC/seco, Rapport annuel de la coopération internationale de la Suisse 2003, Berne, 2004.

seco, Agenda 2010. Pour la réduction de la pauvreté, Berne, 2004.

seco, Année internationale du riz. 2004. Une action du seco en faveur du commerce équitable «Le riz, c'est la vie», Berne, avril 2004.

seco, Coopération au développement. Abrégé du Message sur les mesures de coopération économique et commerciale 2003-2008, Berne, novembre 2002.

seco, La Vie économique, «Les stratégies de lutte contre la pauvreté », thème du mois, 08/2004.

seco, Stratégie 2006, Berne, 2002.

seco/DDC, Bulletin d'information. Coopération avec la Banque mondiale et les institutions financières régionales, Berne, novembre 2004.

seco/DEZA, Die Schweiz und die Weltbank. Leitlinie, Bern, 2004.

\section{ONG}

Communauté de travail Swissaid - Action de Carême - Pain pour le prochain - Helvetas - Caritas EPER, «La stratégie de la Communauté de travail», Dossiers, n 3, octobre 2004.

Niggli Peter, La mondialisation, et après... Quel développement au XXI siècle?, Lausanne, Communauté de travail Swissaid - Action de Carême - Pain pour le prochain - Helvetas - Caritas EPER, 2004.

Oxfam International, Le prix à payer: pourquoi les riches doivent investir maintenant dans la pauvreté (résumé du rapport), décembre 2004. Rapport complet en anglais Paying the Price: Why Rich Countries Must Invest Now in a War on Poverty, Oxford, Oxfam International, <www. oxfam.org/eng/pdfs/pp041206_MDG.pdf>.

Reality of Aid, The Reality of Aid 2004 : An Independant Review of Poverty Reduction and Developement Assistance, Manilla (Philippines); New York (USA), IBON Books ; Zed Books, 2004

\section{SITES INTERNET}

Année internationale du microcrédit: <www.yearofmicrocredit.org $>$.

Année internationale du riz $2004:<w w w . f a o . o r g / r i c e 2004>$.

Comité d'aide au développement (CAD [OCDE]) : <www.oecd.org/dac > .

OCDE, statistiques en ligne: <www.oecd.org/cad/stats/sdienligne>.

Direction du développement et de la coopération (DDC) : <www.deza.admin.ch $>$.

Secrétariat d'Etat à l'économie (seco) : <www.seco.admin.ch>.

Secrétariat d'Etat à l'économie (seco), division Coopération économique au développement: <www. seco-cooperation.ch>.

Communauté de travail des œuvres d'entraide: <www.swisscoalition.ch>.

Oxfam International : <www.oxfam.org/fr>.

Reality of Aid: <www.realityofaid.org $>$. 